\title{
Retrofittable Laser-free kHz to GHz Tunable Pulser for Ultra-fast Electron Microscopy
}

Chunguang Jing ${ }^{1}$, Ao Liu ${ }^{1}$, Eric Montgomery ${ }^{1}$, Yubin Zhao ${ }^{1}$, Hyeokmin Choe ${ }^{1}$, Alexei Kanareykin ${ }^{2}$, June $\mathrm{Lau}^{3}$, Xuewen $\mathrm{Fu}^{4}$ and Yimei $\mathrm{Zhu}^{4}$

${ }^{1}$ Euclid Techlabs, LLC, Bolingbrook, Illinois, United States, ${ }^{2}$ Euclid Techlabs, LLC, Gaithersburg, Maryland, United States, ${ }^{3}$ National Institute of Standards and Technology, Gaithersburg, Maryland, United States, ${ }^{4}$ Brookhaven National Laboratory, Upton, New York, United States

Since its inception [1], the ultrafast transmission electron microscope, (UTEM) which is capable of imaging nanoscale phenomena at ultrafast timescales [2-4], has increasingly gained attractions among researchers. As a result, major TEM vendors have now provided the UTEM option per customers' request. However the entry point is afemtosecond (fs) laser used to pulse electron emission from a photocathode, which presents its own set of barriers to progress: mandatary laser expertise to maintain the laser operation, complete alteration of the electron gun, possible laser heating damage, and a sampling rate much less than $100 \mathrm{MHz}$ - sometimes only $\sim 0.1 \mathrm{MHz}$.

Euclid Techlabs has developed a new method (winner of $R \& D 100$ in 2019) with GHz sampling rates to allow high throughput imaging, enhance signal-to-noise, and produce sharp, fast successions of images and spectra. The device (electron Pulser, see Fig.1, and [5]) challenges laser-based research paradigms by using RF beam-modulators to achieve a pulsed electron beam at 1\% 100\% variable duty cycle with continuously tunable $\mathrm{GHz}$ and $\mathrm{MHz}$ pulse rates, without sacrificing the spatial resolution or function of the original TEM, and it can vary the electrons per pulse, pulse length, and pulse period independently. This allows users to observe dynamic phenomena (also, possibly reduce radiation damage [6]) while maintaining high pulse repetition rate to rapidly accumulate data. This is particularly important for the field of biological imaging and applications for RF sensors, high frequency semiconductors, and magnetic material dynamics. The Pulser retrofits an existing TEM, is compatible with existing detectors, and, eliminates the need for an expensive fs-laser, which could expand usage of UTEM from a few research groups to wide availability.

In 2019, we two Pulsers were installed and are in service. Two pilot experiments were conducted. Figure 1d shows the stroboscopic image of an interdigital MEMS device excited by electromagnetic field [7]. Figure 2 shows preliminary results of the radiation damage study using a pulsed beam produced by our Pulser. In this study, we conducted experiments to compare radiation damage to organic paraffin $\left(\mathrm{C}_{36} \mathrm{H}_{74}\right)$ and biomedical purple membrane samples using picosecond pulsed electrons and continuous random electrons by observing the fading of the diffraction spots from as a function of electron fluence. We always begin with a baseline critical dose experiment by irradiating the biomedical materials with a continuous random electron beam. Then we tested different "pulsed" regimes using the installed electron Pulsers by varying duty cycles. Compared to continuous random beam, our pulsed beam produced up to $2 \mathrm{x}$ less radiation damage to the purple membrane and $2.5 \mathrm{x}$ less damage to the paraffin samples, potentially improving image quality.

Our revolutionary pulser technology will expand scientific knowledge by making ns to ps, on-demand pulse-structured TEM within reach. 


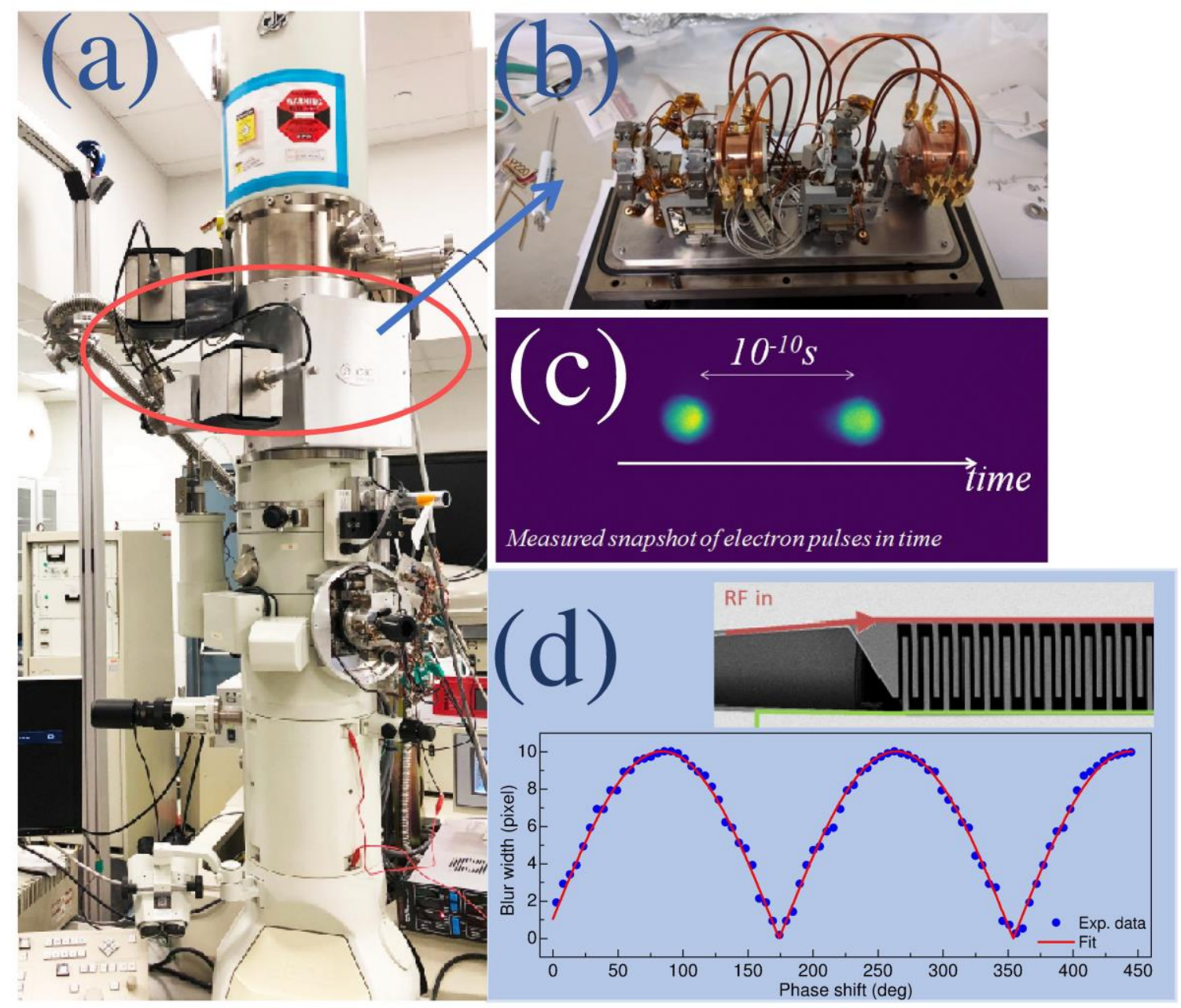

Figure 1. (a) The installed pulser in the JEOL 2100F 200keV TEM at Brookhaven National Laboratory. (b) Front view of the pulser subassembly. (c) The experimental demonstration of temporal resolution displayed as a split beam image. In normal strobe mode, the two beams are coincident. However, in the enhanced modulation diagnostic mode, the downstream demodulation pulser was deliberately de-tuned with respect to the upstream modulation pulser, causing the split-beam. (d) The experimental demonstration of stroboscopic imaging of an interdigital comb: SEM image and results of change of the comb tine width as a function of excitation rf phase [7].

(a)

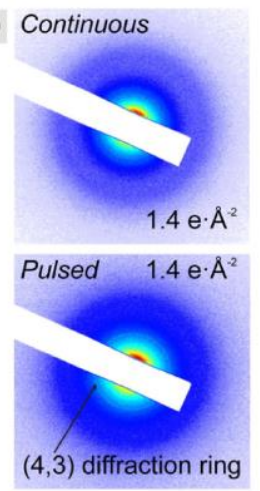

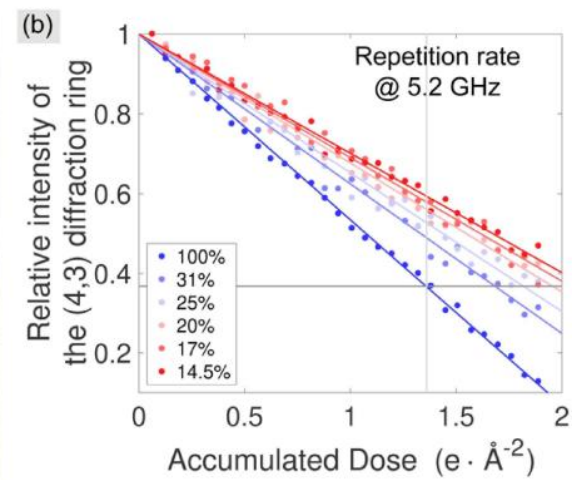

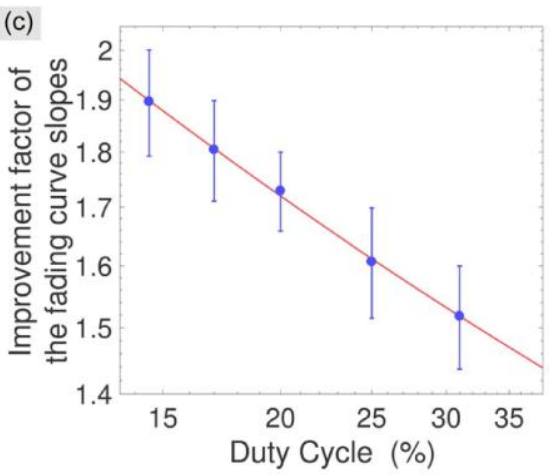


Figure 2. Study of the purple membrane. Comparison of continuous random and pulsed electron beam damage for the same dose rate $(0.014 \mathrm{e} \cdot \AA-2 \cdot \mathrm{s}-1)$. (a) Selected continuous- and pulsed-beam powder diffraction patterns of a purple membrane patches with the $(4,3)$ rings at total accumulated dose $1.8 \mathrm{e} \cdot \AA$ 2. (b) Fading curves from the $(4,3)$ powder rings and linear fits for the electron pulses at different duty cycles. The slope of the linear fit to the continuous electron beam (100\% duty cycle) data is steeper than that of the pulsed beams. Note, because of the unprecedented $10 \mathrm{GHz}$ level of repetition rate of electron pulses, in comparison with continuous random electrons, the difference cannot be explained by sample movement.

References

1. VA Lobastov, R Srinivasan, AH. Zewail, Natl. Acad. Sci. 102 (2005) 7069-7073.

2. Flannigan and A. Zewail, Accounts Chem. Res.,vol. 45, p. 1828 (2012).

3. Zewail, "Four-Dimensional Electron Microscopy", Science, 328 (5975), pp. 187-193 (2010).

4. Kisielowski, C, et al. Discovering hidden material properties of $\mathrm{MgCl} 2$ at atomic resolution with structured temporal electron illumination of picosecond time resolution. Funct. Mater. 2019, 29, 1807818. 5. Jing, et al, Tunable electron beam pulser for picoseconds stroboscopic microscopy in transmission electron microscopes, Ultramicroscopy, 207, December 2019, 112829

6. Elisah J. VandenBussche and David J. Flannigan, Reducing Radiation Damage in Soft Matter with Femtosecond-Timed Single-Electron Packets, Nano Lett. 2019, 19, 6687-6694.

7. June W. Lau, et al, Laser-free GHz stroboscopic TEM: components, system integration, and practical considerations for pump-probe measurements, invited paper, Sci. Inst. 2020, to appear. 